\title{
GLOBAL EXTENSIONS OF SPACETIMES DESCRIBING ASYMPTOTIC FINAL STATES OF BLACK HOLES
}

\author{
István Rácz \\ MTA KFKI Research Institute for Particle and Nuclear Physics \\ H-1525 Budapest 114, P.O.B. 49, Hungary \\ and \\ Robert M. Wald \\ Enrico Fermi Institute and Department of Physics \\ University of Chicago, Chicago IL 60637, USA
}

\begin{abstract}
We consider a globally hyperbolic, stationary spacetime containing a black hole but no white hole. We assume, further, that the event horizon, $\mathcal{N}$, of the black hole is a Killing horizon with compact cross-sections. We prove that if surface gravity is non-zero constant throughout the horizon one can globally extend such a spacetime so that the image of $\mathcal{N}$ is a proper subset of a regular bifurcate Killing horizon in the enlarged spacetime. The necessary and sufficient conditions are given for the extendibility of matter fields to the enlarged spacetime. These conditions are automatically satisfied if the spacetime is static (and, hence " $t$ "-reflection symmetric) or stationary-axisymmetric with " $t-\phi$ " reflection isometry and the matter fields respect the reflection isometry. In addition, we prove that a necessary and sufficient condition for the constancy of the surface gravity on a Killing horizon is that the exterior derivative of the twist of the horizon Killing field vanish on the horizon. As a corollary of this, we recover a result of Carter that constancy of surface gravity holds for any black hole which is static or stationary-axisymmetric with the " $t-\phi$ " reflection isometry. No use of Einstein's equation is made in obtaining any of the above results. Taken together, these results support the view that any spacetime representing the asymptotic final state of a black hole formed by gravitational collapse may be assumed to possess a bifurcate Killing horizon or a Killing horizon with vanishing surface gravity.
\end{abstract}

PACS number: $04.20 \mathrm{Cv}$. 


\section{Introduction}

It is of considerable interest to determine of the possible asymptotic final states of the gravitational collapse of an isolated body. The "cosmic censor hypothesis" conjectures that gravitational collapse always produces a black hole, in such a way that a neighborhood containing the exterior region together with the event horizon of the black hole is globally hyperbolic (see, e.g., the discussion in [1]). In addition, it is widely expected that the asymptotic final state of such a collapse can be represented by a stationary spacetime (i.e., a spacetime possessing a one-parameter group of isometries whose orbits are timelike near infinity). As we shall review in the next section, arguments of Hawking $[2,3]$ and Carter $[4,5]$ show that, in a wide variety of circumstances, the event horizon of a stationary black hole must be a Killing horizon, by which we mean a null hypersurface (i.e., an embedded submanifold of co-dimension one) whose generators coincide with the orbits of a one-parameter group of isometries. Therefore, the study of globally hyperbolic spacetimes with a Killing horizon is of considerable importance with regard to the classification of the possible final states of gravitational collapse.

In a previous paper [6], we analyzed the extendibility of spacetimes with a one-parameter group of isometries possessing a Killing horizon, $\mathcal{N}$, such that the generators on $\mathcal{N}$ are diffeomorphic to $\mathbb{R}$ and that $\mathcal{N}$ admits a smooth cross-section. We showed that whenever the gradient, $\nabla_{a} \kappa$, of the surface gravity, $\kappa$, of $\mathcal{N}$ is non-zero on a generator of $\mathcal{N}$, then that generator terminates in a parallelly propagated curvature singularity, so no extension can exist where $\mathcal{N}$ comprises a portion of a regular, bifurcate Killing horizon (see also [7,8]). On the other hand, if $\kappa$ is constant and nonvanishing on $\mathcal{N}$, we proved that a neighborhood, $\mathcal{U}$, of $\mathcal{N}$ always can be extended so that $\mathcal{N}$ comprises a portion of a bifurcate Killing horizon. However, this analysis did not determine the conditions under which such an extension could be performed globally i.e., such that not merely a neighborhood, $\mathcal{U}$, of the horizon but the entire spacetime can be imbedded into a larger spacetime which possesses a bifurcate Killing horizon. The existence of a global extension would be needed to argue that, without loss of generality, one can assume that $\mathcal{N}$ comprises a portion of a bifurcate Killing horizon.

One might expect that the condition of global hyperbolicity of the original spacetime would suffice, by itself, to ensure the existence of the desired global extension. However, the following example shows that this is not the case, i.e., there exist globally hyperbolic spacetimes possessing a Killing horizon with constant but non-zero surface gravity which can not be extended so that 
the Killing horizon is a portion of a regular, bifurcate horizon.

Example 1.: Start with the 3-dimensional Minkowski spacetime, $\left(\mathbb{R}^{3}, \eta_{a b}\right)$, with Cartesian coordinates, $t, x, y$, and consider the boost isometries about the origin in the $t-x$ plane. Now consider the spacelike surface, $\mathcal{S}$, comprised by all Killing orbits which intersect the line, $x=0$, $t=-|y| / 2$. Let $\Omega$ be a smooth function which is defined on the chronological future, $I^{+}[\mathcal{S}]$, of $\mathcal{S}$, such that it takes the value 1 whenever $t \geq-|x|$, it is "boost-invariant", and for which the curvature scalar of the spacetime, $\left(I^{+}[\mathcal{S}], \Omega^{2} \eta_{a b}\right)$, blows up everywhere 'on' $\mathcal{S} \backslash\left\{(t, x, y) \mid t^{2}-x^{2}=\right.$ 0 and $y=0\}$. An example of such a function, $\Omega$, is

$$
\Omega(t, x, y)= \begin{cases}f\left(2\left(t^{2}-x^{2}\right)^{\frac{1}{2}} /|y|\right), & \text { if } 0 \leq 2\left(t^{2}-x^{2}\right)^{\frac{1}{2}} /|y|<1, t<0, \text { and } y \neq 0 \\ 1, & \text { otherwise }\end{cases}
$$

where $f(z)=1-e^{\left(1-z^{-2}\right)}$, which is well defined for $0 \leq z<1$. The spacetime, $\left(I^{+}[\mathcal{S}], \Omega^{2} \eta_{a b}\right)$, is the desired globally hyperbolic spacetime. The hypersurface $t=x>0$ (as well as the hypersurface $t=-x>0$ ) is Killing horizon, on which the null generator at $y=0$ is geodesically incomplete generator. An open neighborhood of this Killing horizon in the spacetime $\left(I^{+}[\mathcal{S}], \Omega^{2} \eta_{a b}\right)$ is isometric to an open neighborhood of the corresponding Killing horizon in the spacetime, $\left(I^{+}[\mathcal{S}], \eta_{a b}\right)$, so, clearly, the the spacetime $\left(I^{+}[\mathcal{S}], \Omega^{2} \eta_{a b}\right)$ is locally extendible to a spacetime with a bifurcate horizon, in accord with the results of [6]. However, it also is clear that there is no global extension of $\left(I^{+}[\mathcal{S}], \Omega^{2} \eta_{a b}\right)$ to a spacetime with a bifurcate horizon, since there is no way to "put back" the origin so that the generator at $y=0$ will extend through a bifurcation surface. Thus, $\left(I^{+}[\mathcal{S}], \Omega^{2} \eta_{a b}\right)$, is a globally hyperbolic spacetime possessing a non-bifurcate Killing horizon with constant, nonzero surface gravity, which cannot be globally extended to a spacetime possessing a bifurcate Killing horizon.

The difficulty occurring in the above example can be seen to arise from the fact that a portion of the "bottom wedge" (as well as the entire "left wedge") is already present in the original spacetime, and they "get in the way" of any global extension of the portion, $t=x>0$, of the horizon. However, in a spacetime representing the asymptotic final state of a stationary black hole, the "left" and "bottom" wedges would correspond to a white hole region of the spacetime. Such a white hole would not occur in the spacetime describing an actual, physically realistic gravitational collapse, but rather would correspond to a physically irrelevant, "early time" region of the spacetime describing the black hole final state.

In this paper, we will restrict attention to globally hyperbolic, stationary spacetimes (with one asymptotic end) which contain a black hole but no white hole. It should be noted that given 
any globally hyperbolic, stationary spacetime which contains a white hole, the sub-spacetime comprised by $I^{+}\left[\mathcal{I}^{-}\right]$also is stationary and globally hyperbolic but does not contain a white hole. Thus, our assumption that no white hole is present in the spacetime actually involves no loss of generality with regard to the class of spacetimes we consider, but rather merely eliminates a physically irrelevant region of these spacetimes. We shall assume, in addition, that the event horizon, $\mathcal{N}$, of the black hole is a Killing horizon and that $\mathcal{N}$ has compact cross-sections. Our main result is the following: If (and only if) the surface gravity is a non-zero constant on $\mathcal{N}$, one can globally extend the a spacetime so that the image of $\mathcal{N}$ is a proper subset of a regular bifurcate Killing horizon in the enlarged spacetime.

In the next section, some properties of stationary black holes will be reviewed. In addition, we shall give a simple proof that the necessary and sufficient condition of the constancy of $\kappa$ is the vanishing on the horizon of the exterior derivative, $\nabla_{[a} \omega_{b]}$, of the twist, $\omega_{a}\left(\equiv \epsilon_{a b c d} \xi^{b} \nabla^{c} \xi^{d}\right)$, of the horizon Killing field $\xi^{a}$. A corollary of this result yields a result of Carter [5] establishing that if the black hole is static or stationary-axisymmetric with the " $t-\phi$ " reflection isometry, then the surface gravity must be constant over the horizon. Thus, the "zeroth law" of black hole mechanics holds in this context without the imposition of any field equations.

Some properties of the space of Killing orbits are established in section 3, in preparation for the proof of our main result on global extensions given in section 4 . The extendibility of matter fields is analyzed in section 5 .

Finally, we emphasize that, as in our previous paper [6], no use of Einstein's (or any other) field equation is made anywhere in our analysis. Furthermore, although for definiteness we treat the case of a 4-dimensional spacetime, all of our results generalize straightforwardly to any spacetime dimension $n \geq 2$. Similarly, although for simplicity, we shall assume that the black hole event horizon, $\mathcal{N}$, is connected our results generalize straightforwardly to the case where $\mathcal{N}$ possesses disconnected components.

\section{Stationary black holes and the Zeroth Law}

In this section, we shall give a simple proof of a generalization of a result of Carter on the constancy of the surface gravity of static and stationary-axisymmetric black holes, and we shall specify the precise class of spacetimes we shall consider in the following sections.

We begin by briefly recalling the arguments that the event horizon of a stationary black 
hole must be a Killing horizon. Actually, there are two complementary approaches which lead to this conclusion. The argument of Hawking [2,3] assumes that Einstein's equation holds with matter satisfying suitable hyperbolic equations and the dominant energy condition. Moreover, it assumes that the spacetime is analytic. By consideration of the null initial value formulation (with one of the two intersecting null hypersurfaces taken to be the event horizon of the black hole), it is then shown that the initial data must be invariant under the action of a one parameter group whose orbits on the event horizon coincide with its null geodesic generators. By uniqueness of the Cauchy evolution together with analyticity, it then follows that the spacetime possesses a one-parameter group of isometries, with Killing field normal to the event horizon, i.e., that the event horizon is a Killing horizon. Note that if the stationary Killing field fails to be normal to the event horizon, this argument shows that there must exist an additional Killing field. Further arguments establish that, in this case, a linear combination of the stationary Killing field and the Killing field orthogonal to the horizon must have closed orbits, so that the spacetime is axisymmetric as well as stationary.

The argument of Carter $[4,5]$ assumes that the black hole is either static or is stationaryaxisymmetric with a " $t-\phi$ " reflection isometry. In the static case, it then is shown that the black hole event horizon must coincide with (a portion of) the "staticity limit", defined to be the boundary of the region where the static Killing field is timelike. Similarly, in the stationary-axisymmetric case, the black hole event horizon must coincide with (a portion of) the "circularity limit", defined to be the boundary of the region where there exists a linear combination of the stationary and axial Killing fields which is timelike. In either case, it then is shown that the event horizon must be a Killing horizon. In the static case, the static Killing field itself must be orthogonal to the event horizon. In the stationary-axisymmetric case, some constant linear combination of the stationary and axial Killing fields must be orthogonal to the horizon. Note that although this argument makes considerably stronger assumptions about the symmetries of the black hole spacetime compared with the argument of the previous paragraph, Einstein's equation is not used, so the argument is applicable to much more general theories of gravity.

Next, we recall the surface gravity, $\kappa$, of a Killing horizon, $\mathcal{N}$, with Killing field $\xi^{a}$ is defined on $\mathcal{N}$ by the equation

$$
\frac{1}{2} \nabla^{a}\left(\xi^{b} \xi_{b}\right)=-\kappa \xi^{a}
$$


It follows immediately that $\kappa$ is constant along the orbits of $\xi^{a}$. Our main new result of this section is the following:

Theorem 2.1: Let $\mathcal{N}$ be a connected Killing horizon, with Killing field $\xi^{a}$. Then the surface gravity, $\kappa$, is constant on $\mathcal{N}$ if and only if the exterior derivative of the twist form field, $\omega_{a}$, is zero on the horizon, i.e.,

$$
\left.\nabla_{[a} \omega_{b]}\right|_{\mathcal{N}}=0
$$

where $\omega_{a}$ is defined by $\omega_{a}=\epsilon_{a b c d} \xi^{b} \nabla^{c} \xi^{d}$.

Proof: By a standard relation satisfied by the surface gravity (see, e.g., eq. (12.5.30) of [1]), we have

$$
\xi_{[a} \nabla_{b]} \kappa=-\xi_{[a} R_{b]}^{e} \xi_{e} .
$$

On the other hand, by a standard identity satisfied by the twist (see, e.g., eq. (7.1.15) of [1]), we have

$$
\nabla_{[a} \omega_{b]}=-\epsilon_{a b c d} \xi^{[c} R^{d]}{ }_{e} \xi^{e}
$$

Thus, on the horizon, we have

$$
\xi_{[a} \nabla_{b]} \kappa=-\frac{1}{4} \epsilon_{a b c d} \nabla^{[c} \omega^{d]}
$$

from which the theorem follows immediately.

This theorem has, as a consequence, the following corollary, which expresses the essential content of a result previously obtained by Carter (see theorem 8 of [5]; see also Heusler [9]):

Corollary 2.2: Let $\mathcal{N}$ be a connected Killing horizon, with Killing field $\xi^{a}$. Then, (i) If $\xi^{a}$ is hypersurface orthogonal, then $\kappa$ must be constant throughout the horizon. In particular, $\kappa$ is constant on the horizon of any static black hole. (ii) If there exists a Killing field, $\psi^{a}$, on the spacetime which is linearly independent of $\xi^{a}$, commutes with $\xi^{a}$, and on the horizon satisfies $\nabla_{a}\left(\psi^{b} \omega_{b}\right)=0$, then $\kappa$ is constant on $\mathcal{N}$. In particular, $\kappa$ is constant on the horizon of any stationary-axisymmetric black hole possessing the " $t-\phi$ " reflection isometry.

Proof: The first claim of (i) is trivial, since $\omega_{a}=0$ everywhere in the hypersurface orthogonal case. The second claim of (i) follows from the fact [5] (reviewed above) that the event horizon 
of a static black hole is a Killing horizon, with $\xi^{a}$ equal to the static (and, thus, hypersurface orthogonal) Killing field.

To prove the first claim of (ii), we assume the contrary. Then there exists an open subset, $\mathcal{O}$, of $\mathcal{N}$ such that in $\mathcal{O}$ we have $\xi_{[a} \nabla_{b]} \kappa \neq 0$. Clearly then, without loss of generality, we may assume that $\kappa \neq 0$ in $\mathcal{O}$. Since $\psi^{a}$ is a Killing field which commutes with $\xi^{a}$, we have

$$
0=\mathcal{L}_{\psi}\left(\xi^{a} \xi_{a}\right)=-2 \kappa \psi^{a} \xi_{a}
$$

so $\psi^{a}$ is tangential to $\mathcal{N}$ in $\mathcal{O}$. Now, if $\psi^{a}$ were proportional to $\xi^{a}$ in $\mathcal{O}$ - i.e., if $\psi^{a}=f \xi^{a}$ in $\mathcal{O}$ - then the commutativity of $\psi^{a}$ and $\xi^{a}$ implies that $\xi^{a} \nabla_{a} f=0$ in $\mathcal{O}$. On the other hand, since $\psi^{a} \psi_{a}=0$ in $\mathcal{O}$, it follows that $\mathcal{O}$ also is (a portion of) a Killing horizon with respect to $\psi^{a}$, with surface gravity with respect to $\psi^{a}$ given by $\tilde{\kappa}=f \kappa$. Application of eq. (3) to both $\kappa$ and $\tilde{\kappa}$ then implies that $\xi_{[a} \nabla_{b]} f=0$. Thus, we have $\nabla_{a} f=0$ in $\mathcal{O}$, which, in turn, implies that $\psi^{a}$ and $\xi^{a}$ are linearly dependent as Killing fields, contrary to our hypothesis. This shows that there must exist a point $p \in \mathcal{O}$ where $\psi^{a}$ is strictly spacelike. Now, since $\psi^{a}$ is a Killing field which commutes with $\xi^{a}$, it follows immediately from eq. (1) that $\psi^{a} \nabla_{a} \kappa=0$ at $p$. On the other hand, from eq. (5) we obtain

$$
\epsilon^{e f a b} \psi_{f} \xi_{a} \nabla_{b} \kappa=\psi_{f} \nabla^{[e} \omega^{f]}
$$

But, since $\omega_{a}$ vanishes on $\mathcal{N}$ (since $\xi^{a}$ is hypersurface orthogonal on $\mathcal{N}$ ) and since $\mathcal{L}_{\psi} \omega_{a}=0$ everywhere (since $\psi^{a}$ is a Killing field which commutes with $\xi^{a}$ ), the right side of eq. (7) can be rewritten as

$$
\psi_{f} \nabla^{[e} \omega^{f]}=\frac{1}{2} \nabla^{e}\left(\psi_{f} \omega^{f}\right)
$$

which vanishes by hypothesis. Thus, both $\epsilon^{e f a b} \psi_{f} \xi_{a} \nabla_{b} \kappa$ and $\psi^{a} \nabla_{a} \kappa$ vanish at $p$, which implies that $\xi_{[a} \nabla_{b]} \kappa=0$ at $p$, in contradiction to our hypothesis. This establishes the first claim of (ii).

Finally, the second claim of (ii) follows from the fact [5] (reviewed above) that the event horizon of a stationary-axisymmetric black hole with " $t-\phi$ " reflection symmetry is a Killing horizon with respect to some constant linear combination, $\xi^{a}$, of the stationary and axial Killing fields. If we choose $\psi^{a}$ to be the axial Killing field, then the Frobenius integrability condition for the " $t-\phi$ " reflection symmetry requires that $\psi^{a} \omega_{a}=0$ throughout the spacetime, so the hypothesis of the first claim of (ii) holds. 
We emphasize that in the derivation of the above results, no restriction has been imposed on the causality structure of the spacetime or on the topological properties of the Killing horizon (except for our simplifying assumption that $\mathcal{N}$ is connected). In particular, these results are valid for any metric theory of gravity, including theories with torsion (with the understanding that in our formulas, $\nabla_{a}$ always denotes the torsion free, metric compatible derivative operator).

We conclude this section by giving a mathematically precise specification of the class of spacetimes, $\left(M, g_{a b}\right)$, which will be considered in the remainder of this paper. We require that $\left(M, g_{a b}\right)$ be a smooth, globally hyperbolic spacetime, possessing a smooth spacelike Cauchy surface $\Sigma$ which can be expressed in the form $\Sigma=\Sigma_{e n d} \cup \Sigma^{\prime}$ so that $\left(M, g_{a b}\right)$ is $(k, \alpha)$-asymptotically stationary with respect to the single asymptotically flat "end" $\Sigma_{e n d}$, as specified in definition 2.1 of [10]. However, it should be noted that the precise asymptotic flatness conditions on $\Sigma_{\text {end }}$ given in that definition will not be of great importance here, and could be significantly weakened or modified. We denote by $\phi_{u}$ the one-parameter group of isometries whose orbits are timelike on $\Sigma_{\text {end }}$, and denote its associated Killing field by $t^{a}$. We define $M_{\text {end }}$ to be the orbit of $\Sigma_{\text {end }}$ under these isometries,

$$
M_{\text {end }}=\phi\left\{\Sigma_{\text {end }}\right\}
$$

The black hole region, $\mathcal{B}$, of $M$ is defined to be the complement of $I^{-}\left[M_{\text {end }}\right]$, and the white hole region, $\mathcal{W}$, is defined to be the complement of $I^{+}\left[M_{\text {end }}\right]$. As discussed in the introduction, we shall assume that $\mathcal{B} \neq \emptyset$ but $\mathcal{W}=\emptyset$, so that

$$
M=I^{+}\left[M_{\text {end }}\right] .
$$

The future event horizon of the black hole is defined by

$$
\mathcal{N}=\partial I^{-}\left[M_{\text {end }}\right]
$$

As discussed above, we shall assume that $\mathcal{N}$ is a Killing horizon, i.e., that there exists a oneparameter group of isometries $\chi_{u}$ (possibly different from $\phi_{u}$ ) with Killing field $\xi^{a}$ normal to $\mathcal{N}$. We shall assume that the intersection of $\mathcal{N}$ with $\Sigma$ is compact. In addition, for simplicity, we assume that $\mathcal{N}$ is connected. By reversing the sign of $\xi^{a}$ if necessary, we may assume that the orbits of $\xi^{a}$ are future directed on $\mathcal{N}$. If $\xi^{a} \neq t^{a}$, it follows from our asymptotic conditions that a linear combination of $\xi^{a}$ and $t^{a}$ has closed orbits, i.e., that the spacetime is stationary 
and axisymmetric. In particular - whether or not $\xi^{a}=t^{a}-$ it follows that $\left(M, g_{a b}\right)$ is $(k, \alpha)$ asymptotically stationary-rotating with respect to $\xi^{a}$ (see the definition of Appendix A of [10]); in particular, the orbits of $\xi^{a}$ in $M_{e n d}$ are future oriented, i.e., for each $p \in M_{e n d}$ there exists an increasing sequence $\left\{u_{i}\right\}$ such that $\chi_{u_{i+1}}(p) \in I^{+}\left(\chi_{u_{i}}(p)\right)$. We note that our results could be generalized straightforwardly to spacetimes which are merely stationary-rotating with respect to the Killing field, $\xi^{a}$, which is normal to the horizon, without the need to assume the existence of the asymptotically stationary Killing field $t^{a}$.

\section{Space of Killing orbits}

In this section we shall consider the class of spacetimes, $\left(M, g_{a b}\right)$, specified at the end of the previous section and we will establish some properties of the space of Killing orbits which will be needed to perform our global extension. Our first result is the following:

Lemma 3.1: Let $\left(M, g_{a b}\right)$ be a spacetime in the class specified at the end of the previous section. Then no zero of $\xi^{a}$ exists in $M$.

Proof: Suppose, on the contrary, that $\left.\xi^{a}\right|_{p}=0$ for some $p \in M$. Since $p$ is invariant under the isometries, it follows from lemma 3.1 of [10] that either $I^{-}(p) \cap M_{\text {end }}=\emptyset$ or $I^{-}(p) \supset M_{\text {end }}$. The first possibility is excluded by our assumption that $M=I^{+}\left[M_{e n d}\right]$. However, the second possibility would imply that $I^{-}(p) \supset \Sigma_{\text {end }}$, which contradicts the fact that $J^{-}(p)$ has to intersect any Cauchy surface $\Sigma$ in a compact set.

Since, in particular, the horizon, $\mathcal{N}$, contains no fixed point of $\chi_{u}$, any smooth spacelike Cauchy surface, $\mathcal{C}$, intersects $\mathcal{N}$ in a global cross-section, $\sigma$. This fact combined with the fact that for globally hyperbolic spacetimes the Killing orbits lying on $\mathcal{N}$ are diffeomorphic to $\mathbb{R}$ implies that condition 2.1 of Ref. [6] is automatically satisfied for the class of spacetimes considered here.

Let $\mathcal{C}$ be an arbitrary smooth, spacelike Cauchy surface, and let $\sigma$ denote the intersection, $\mathcal{N} \cap \mathcal{C}$, of the Killing horizon and the Cauchy surface. Then $\sigma$ is a smooth, embedded submanifold of $\mathcal{C}$, which, by our assumption above, must be compact. There are two families of null geodesics the members of which are orthogonal to $\sigma$. One of these families generates the Killing horizon, $\mathcal{N}$, while the members of the other congruence determine - at least in a sufficiently small neighborhood of $\sigma-$ a smooth null hypersurface, $P$. The next lemma shows that in a 
sufficiently small neighborhood of $\sigma, P$ comprises a portion of boundary of the future and past of $\sigma$.

Lemma 3.2: There exists an open neighborhood, $\mathcal{U}$, of $\sigma$ in $M$ such that $\left[P \cap J^{+}[\sigma] \cap \mathcal{U}\right] \subset$ $\partial J^{+}[\sigma]$, and, similarly, $\left[P \cap J^{-}[\sigma] \cap \mathcal{U}\right] \subset \partial J^{-}[\sigma]$

Proof: It suffices to prove the first claim, since the second claim follows by interchanging futures and pasts. Consider the map from the normal bundle of $\sigma$ into $M$ which assigns to each $\left(s, n^{a}\right)$ in the normal bundle (so that $s \in \sigma$ and $n^{a}$ is in the tangent space at $s$ and normal to $\sigma$ ) the point in $M$ lying at a unit affine parameter along the geodesic determined by $\left(s, n^{a}\right)$. Then this map is smooth, and, by the implicit function theorem, there exists a neighborhood $\mathcal{U}_{1}$ of $\sigma$ such that this map is one-to-one and onto. Let $\mathcal{U}_{2}$ be a causal normal neighborhood of $\mathcal{C}$ (see lemma 2.2 of [8]) and let $\mathcal{U}=\mathcal{U}_{1} \cap \mathcal{U}_{2}$. Let $p \in P \cap J^{+}[\sigma] \cap \mathcal{U}$. For $s \in J^{-}(p) \cap \sigma$ define $\tau_{p}(s)$ to be the length (defined to be $\geq 0$ ) of the unique causal geodesic connecting $p$ with $s$; for $s \in \sigma$ but $s \notin J^{-}(p)$, define $\tau_{p}(s)$ to be 0 . Then $\tau_{p}(s)$ is a continuous function of $s$. Since $J^{-}(p)$ is closed in a globally hyperbolic spacetime, it follows that $J^{-}(p) \cap \sigma$ is compact and non-empty, so $\tau_{p}(s)$ must achieve a maximum at a point $s_{0} \in \sigma$. It follows that $p$ must be connected to $s_{0}$ by a causal geodesic which is orthogonal to $\sigma$. However, since $p \in \mathcal{U}_{1}$, there is a unique geodesic from $p$ to $\sigma$ which is orthogonal to $\sigma$, and since $p \in P$, this geodesic is a null geodesic. Hence, $\tau_{p}(s)=0$ for all $s \in \sigma$, i.e., $p$ cannot be connected to any point of $\sigma$ by a timelike geodesic. It follows that $p \in \partial J^{+}[\sigma]$, as we desired to show.

We now define $\mathcal{P}=P \cap \mathcal{U}$. The next lemma shows that no timelike curve can start on $\mathcal{P}$ and return to $\mathcal{P}$. Similarly, Killing orbits are shown to intersect $\mathcal{P}$ precisely once.

Lemma 3.3: a) $\mathcal{P}$ is an achronal hypersurface.

b) Any Killing orbit starting on $\mathcal{P}$ never intersects $\mathcal{P}$ again.

Proof: a) Let $p, q \in \mathcal{P}$. We wish to show that there does not exist a timelike curve, $\gamma$, from $p$ to $q$. If $p, q \in \partial J^{+}[\sigma]$ or if $p, q \in \partial J^{-}[\sigma]$, the result follows immediately from the previous lemma together with the achronality of causal boundaries. Thus, we need only consider the case $p \in \partial J^{-}[\sigma] \cap \mathcal{P}$ and $q \in \partial J^{+}[\sigma] \cap \mathcal{P}$. In this case, any timelike curve $\gamma$ from $p$ to $q$ must intersect $\mathcal{C}$ at a point $r$. However, if $r \in \mathcal{B}$ we would obtain a contradiction with $p \in \partial J^{-}[\sigma] \cap \mathcal{P}$, since $\left[\partial J^{-}[\sigma] \cap \mathcal{P}\right] \subset \partial J^{-}[\mathcal{C} \cap \mathcal{B}]$. Similarly, if $r \notin \mathcal{B}$, we would contradict $q \in \partial J^{+}[\sigma] \cap \mathcal{P}$.

b) We wish to show that for any $p \in \mathcal{P}$ there does not exist a $u>0$ such that $\chi_{u}(p) \in \mathcal{P}$. 
If $p \in \sigma$, the Killing orbit through $p$ is a (future-directed) null geodesic generator of $\mathcal{N}$, and $\chi_{u}(p) \in I^{+}[\mathcal{C}]$ for all $u>0$. Since $\mathcal{N} \cap \mathcal{P}=\sigma$, it follows that $\chi_{u}(p) \notin \mathcal{P}$. On the other hand, if $p \in J^{+}[\sigma] \backslash \sigma$, then there exists a future-directed null geodesic $\lambda$ from a point $s \in \sigma$ to $p$. Applying the isometry $\chi_{u}$ to this statement, we find that there is a future-directed null geodesic joining $\chi_{u}(s)$ to $\chi_{u}(p)$. Hence, $s$ may be joined to $\chi_{u}(p)$ by a (future-directed) broken null geodesic. It follows that $\chi_{u}(p) \in I^{+}[\sigma]$ and, hence, by part (a) of this lemma we have $\chi_{u}(p) \notin \mathcal{P}$. The remaining case $p \in J^{-}[\sigma] \backslash \sigma$ follows similarly.

The above lemma shows that the isometry invariant open neighborhood, $\mathcal{O}_{\mathcal{N}}=\chi\{\mathcal{P}\}$, of the horizon, $\mathcal{N}$ possesses the structure of a trivial principal fibre bundle, with structure group $\mathbb{R}$. Nevertheless, it remains possible that the Killing orbits in $\mathcal{O}_{\mathcal{N}}$ could come arbitrarily close to other Killing orbits in $M$ which do not intersect $\partial \mathcal{P}$. (Note that if that occurred, the space of Killing orbits in $M$ would fail to have Hausdorff topology.) If this were to happen, these additional Killing orbits could "get in the way" of an attempted global extension of $M$. The following provides a relevant example of a globally hyperbolic spacetime possessing a Killing horizon where Killing orbits in $\mathcal{O}_{\mathcal{N}}$ come arbitrarily close to Killing orbits which do not intersect the closure of $\mathcal{P}$.

Example 2: Let $\left(M, g_{a b}\right)$ be Minkowski spacetime with the "bottom wedge" $t \leq-|x|$ removed. The resulting spacetime is globally hyperbolic and possesses a one parameter group of isometries generated by the boost Killing field $\xi=t \frac{\partial}{\partial x}+x \frac{\partial}{\partial t}$, which is nowhere vanishing on $M$. Furthermore, the hypersurface $t=x$ is a Killing horizon with respect to $\xi^{a}$. Nevertheless, for any choice of $\mathcal{P}$, the closure of the set $\mathcal{O}_{\mathcal{N}}=\chi\{\mathcal{P}\}$ includes all of the Killing orbits with $t=-x$.

The next lemma shows that the type of behavior exhibited in the above example cannot occur for the class of spacetimes considered here.

Lemma 3.4: Let $\mathcal{N}, \sigma$ and $\mathcal{P}$ be defined as above. Let $\mathcal{P}^{*}$ to be an open subset of $\mathcal{P}$ with compact closure in $\mathcal{P}$. Then the boundary (in $M$ ) of the set $\chi\left\{\mathcal{P}^{*}\right\}$ is generated by orbits meeting $\mathcal{P}$ at the boundary of $\mathcal{P}^{*}$.

Proof: Suppose on the contrary that there exists $q \in \partial\left[\chi\left\{\mathcal{P}^{*}\right\}\right] \backslash \chi\left\{\partial \mathcal{P}^{*}\right\}$ in $M$. The statement that $q \in \partial\left[\chi\left\{\mathcal{P}^{*}\right\}\right]$ implies that there exists a sequence $\left\{p_{i}\right\}$ in $\mathcal{P}$ and a sequence of real numbers $\left\{u_{i}\right\}$ such that the sequence $\left\{q_{i}=\chi_{u_{i}}\left(p_{i}\right)\right\}$ converges to $q$. Since $\mathcal{P}^{*}$ has compact closure in $\mathcal{P}$ there must exist a subsequence of the $\left\{p_{i}\right\}$ converging to a point $p$ in $\overline{\mathcal{P}^{*}} \subset \mathcal{P}$. Passing to 
this subsequence, we note that if the sequence $\left\{u_{i}\right\}$ had an accumulation point, $u$, then we would have $q=\chi_{u}(p)$, in contradiction to our hypothesis. It follows that we must have either $u_{i} \rightarrow-\infty$ or $u_{i} \rightarrow+\infty$ as $i \rightarrow \infty$. Consider, first, the case $u_{i} \rightarrow-\infty$. Let $p^{\prime} \in I^{+}(p)$ and let $q^{\prime} \in I^{-}(q)$. Then there exists an integer $\bar{i}$ such that for all $i>\bar{i}$ we have $p_{i} \in I^{-}\left(p^{\prime}\right)$ and $q_{i} \in I^{+}\left(q^{\prime}\right)$. However, we have $q^{\prime} \in M=I^{+}\left[M_{\text {end }}\right]$, so there exists $r \in M_{\text {end }}$ such that $r \in I^{-}\left(q^{\prime}\right)$ and, hence, $r \in I^{-}\left(q_{i}\right)$ for all $i>\bar{i}$. Applying the isometry $\chi_{-u_{i}}$ to this statement, we find that $\chi_{-u_{i}}(r) \in I^{-}\left(p_{i}\right)$ and, hence, $\chi_{-u_{i}}(r) \in I^{-}\left(p^{\prime}\right)$ for all $i>\bar{i}$. Since the Killing orbits are future oriented in $M_{e n d}$, this implies that $I^{-}\left(p^{\prime}\right)$ contains the entire Killing orbit through $r$, which, in turn, implies that $I^{-}\left(p^{\prime}\right) \supset M_{\text {end }}$. However, as in the proof of Lemma 3.1, this contradicts the fact that, on account of global hyperbolicity, $J^{-}\left(p^{\prime}\right) \cap \Sigma$ must be compact.

The proof for the case $u_{i} \rightarrow+\infty$ follows by interchanging the roles of $p$ and $q$ in the above argument.

\section{Global extensions}

The global extension of the class of black hole spacetimes specified at the end of section 2 will be based on the use of so-called Eddington-Finkelstein-type (EF) coordinates. These coordinates were introduced in [6] in a neighborhood of the Killing horizon, $\mathcal{N}$. We begin by reviewing the construction of these $\mathrm{EF}$ coordinates.

Let $\sigma$ be global cross-section of $\mathcal{N}$ obtained by intersecting $\mathcal{N}$ with a Cauchy surface $\mathcal{C}$ as above. Consider, now, an open subset, $\tilde{\sigma}$, of $\sigma$ on which local coordinates $x^{1}, x^{2}$ can be introduced. Denote by $\tilde{\mathcal{N}}$ and $\tilde{\mathcal{P}}$ the portions of $\mathcal{N}$ and $\mathcal{P}$, respectively, generated by null geodesics which intersect $\tilde{\sigma}$. First, we extend the coordinates $x^{1}, x^{2}$ on $\tilde{\sigma}$ to $\tilde{\mathcal{P}}$ by keeping them constant on the null geodesic generators of $\tilde{\mathcal{P}}$. Consider, now, the unique null vector field, $\eta^{a}$, on $\tilde{\sigma}$ satisfying $\eta^{a} \xi_{a}=1$ and $\eta^{a} X_{a}=0$ for all $X^{a}$ which are tangent to $\tilde{\sigma}$. We define the function $r$ on $\tilde{\mathcal{P}}$ to be the value of the affine parameter along the null geodesic generators of $\tilde{\mathcal{P}}$ starting on $\tilde{\sigma}$ (with $\left.r\right|_{\tilde{\sigma}}=0$ ) with tangent $\eta^{a}$. Then $\left(r, x^{1}, x^{2}\right)$ comprise coordinates on $\tilde{\mathcal{P}}$. We extend the functions, $r, x^{1}, x^{2}$, from $\tilde{\mathcal{P}}$ to $\mathcal{O}_{\tilde{\mathcal{N}}} \equiv \chi\{\tilde{\mathcal{P}}\}$ by requiring their values to be constant along the Killing orbits through $\tilde{\mathcal{P}}$. Next we define the function $u$ on $\mathcal{O}_{\tilde{\mathcal{N}}}$ by the conditions $\xi^{a} \nabla_{a} u=1$ and $u=0$ on $\tilde{\mathcal{P}}$. Then $\left(u, r, x^{1}, x^{2}\right)$ yields the desired EF coordinate system on $\mathcal{O}_{\tilde{\mathcal{N}}}$.

It follows [6] that the spacetime metric in $\mathcal{O}_{\tilde{\mathcal{N}}}$ takes the form

$$
d s^{2}=-F d u^{2}+2 d u d r+2 g_{u \alpha} d u d x^{\alpha}+g_{\alpha \beta} d x^{\alpha} d x^{\beta}
$$


where $F=-\xi^{a} \xi_{a}, g_{u \alpha}=\xi_{a}\left(\frac{\partial}{\partial x^{\alpha}}\right)^{a}$, and $g_{\alpha \beta}$ are smooth functions of $r, x^{1}, x^{2}$ in $\mathcal{O}_{\tilde{\mathcal{N}}}$ such that $F$ and $g_{u \alpha}$ vanish on $\tilde{\sigma}$ and $g_{\alpha \beta}$ is a positive definite $2 \times 2$-matrix. (The Greek indices take the values 1,2 .) As shown in [6], if the surface gravity, $\kappa$, of $\tilde{\mathcal{N}}$ is constant and nonvanishing, then Kruskal-type coordinates $\left(U, V, x^{1}, x^{2}\right)$ can be defined in an open neighborhood, $\tilde{\mathcal{O}} \subset \mathcal{O}_{\tilde{\mathcal{N}}}$ of $\tilde{\mathcal{N}}$ - comprised by the points $\mathcal{O}_{\tilde{\mathcal{N}}}$ for which $|U V|<\tilde{\varepsilon}\left(x^{1}, x^{2}\right)$ for some suitably chosen positive function $\tilde{\varepsilon}\left(x^{1}, x^{2}\right)-$ by

$$
\begin{gathered}
U=e^{\kappa u} \\
V=-r e^{-\kappa u} \exp \left[2 \kappa \int_{0}^{r} g\left(r^{\prime}, x^{1}, x^{2}\right) d r^{\prime}\right],
\end{gathered}
$$

where

$$
g \equiv \frac{1}{F}-\frac{1}{2 r \kappa}
$$

In the Kruskal coordinates, the spacetime metric, $g_{a b}$, in $\tilde{\mathcal{O}}$ takes the form [6]

$$
d s^{2}=G d U d V+V H_{\alpha} d U d x^{\alpha}+g_{\alpha \beta} d x^{\alpha} d x^{\beta},
$$

Here $G, H_{\alpha}$, and $g_{\alpha \beta}$ are smooth functions of the three quantities $U V, x^{1}, x^{2}$. The precise range of the Kruskal type coordinates in $\tilde{\mathcal{O}}$ is given by the inequality $|U V|<\tilde{\varepsilon}\left(x^{1}, x^{2}\right)$ together with the restrictions on the original range of the coordinates $\left(x^{1}, x^{2}\right)$, and the inequality $U>0$. Note that, by construction, $\tilde{\mathcal{O}}$ is comprised by complete Killing orbits.

As in [6], we can construct a local extension of the spacetime to one which contains a bifurcate Killing horizon as follows: Let $\left\{\tilde{\sigma}_{(i)}\right\}$ be a family of charts covering $\sigma=\mathcal{N} \cap \mathcal{C}$. Since $\sigma$ is compact, we may take $\left\{\sigma_{(i)}\right\}$ be a finite collection of charts. For each $i$, we introduce EF coordinates as above, and let $\left(\tilde{M}_{(i)}, \tilde{g}_{(i)} a b\right)$ be the spacetime defined by eq. (16), with the range of the coordinates given by the original range of the EF coordinates $\left(x^{1}, x^{2}\right)$ together with the inequality $|U V|<\tilde{\varepsilon}_{(i)}\left(x^{1}, x^{2}\right)$. In other words, the spacetime manifold is $\tilde{M}_{(i)}=\tilde{\sigma}_{(i)} \times \mathbb{R}^{2}$ with metric given by (16), but with the restriction $U>0$ now dropped. As in [6], we may "patch together" the spacetimes $\left(\tilde{M}_{(i)}, \tilde{g}_{(i)} a b\right)$ to obtain a new spacetime $\left(\tilde{M}, \tilde{g}_{a b}\right)$ by taking the union of the $\left(\tilde{M}_{(i)}, \tilde{g}_{(i)} a b\right)$ and then identifying the points labeled by $\left(U_{(i)}, V_{(i)}, x_{(i)}^{1}, x_{(i)}^{2}\right)$ and $\left(U_{(j)}, V_{(j)}, x_{(j)}^{1}, x_{(j)}^{2}\right)$ if and only if $\left(x_{(i)}^{1}, x_{(i)}^{2}\right)$ corresponds to the same point of $\sigma$ as $\left(x_{(j)}^{1}, x_{(j)}^{2}\right)$ and $U_{(i)}=U_{(j)}, V_{(i)}=V_{(j)}$ (see the bottom of P. 2651 of [6] for details). Note that the functions $U$ and $V$ are then globally well defined in $\left(\tilde{M}, \tilde{g}_{a b}\right)$. It may be verified straightforwardly (see $[6])$ that $\left(\tilde{M}, \tilde{g}_{a b}\right)$ is an extension of the spacetime $\left(\mathcal{U}, g_{a b}\right)$, where $\mathcal{U}=\bigcup_{i} \tilde{\mathcal{O}}_{(i)} \subset M$. Here the 
isometric embedding $\phi: \mathcal{U} \rightarrow \tilde{M}$ arises from the maps $\tilde{\phi}_{(i)}: \tilde{\mathcal{O}}_{(i)} \rightarrow \tilde{M}_{(i)}$ which take each point of $\tilde{\mathcal{O}}_{(i)}$ into the point in $\tilde{M}_{(i)}$ having the same values of the coordinates $\left(U_{(i)}, V_{(i)}, x_{(i)}^{1}, x_{(i)}^{2}\right)$. It also is straightforward to verify that the hypersurfaces defined by $U=0$ and $V=0$ comprise a bifurcate Killing horizon, $\mathcal{H}$, in $\tilde{M}$ and that the spacetime $\left(\tilde{M}, \tilde{g}_{a b}\right)$ possesses a reflection isometry defined by $U \rightarrow-U, V \rightarrow-V$ about the bifurcation surface, $S$, given by $U=V=0$. Finally, we note that the image of $\mathcal{N}$ under $\phi$ comprises the portion of $\mathcal{H}$ defined by $V=0$, $U>0$.

Note that, using the isometric imbedding, $\phi$, one can show that the Killing orbits generating $\mathcal{N}$ must be geodesically incomplete in the past rather than the future; equivalently, the surface gravity, $\kappa$ defined by eq. (1) must be positive rather than negative. Namely, suppose, on the contrary, that the orbits on $\mathcal{N}$ were future incomplete. Then the hypersurfaces of constant $U V$ sufficiently close to $\mathcal{N}$ would be spacelike in $I^{-}[\mathcal{N}]$, and, consequently, in this region $U V$ would have to decrease along all future-directed causal curves. From this it would follow that for any $p \in I^{-}[\mathcal{N}]$ sufficiently close to $\mathcal{N}$, the image under $\phi$ of any future-directed null geodesic starting at $p$ would remain in $\tilde{M}$ at least until reaching $\mathcal{H}$. However, this would contradict the fact that some future-directed null geodesics from $p$ must reach $M_{e x t}$. Thus, the horizon, $\mathcal{N}$, of $\left(M, g_{a b}\right)$ cannot be "upside down" with respect to the usual orientation of the horizon occurring, e.g., in the Schwarzschild solution.

The above local extension will now be "globalized" with the help of lemma 3.4 together with the following general lemma:

Lemma 4.1: Let $\left(M, g_{a b}\right)$ be an $n$-dimensional spacetime without boundary, and let $\left(M^{\prime}, g^{\prime}{ }_{a b}\right)$ be an $n$-dimensional spacetime with boundary $\partial M^{\prime}$. Let $\mathcal{O}^{\prime}$ be an $n$-dimensional submanifold with boundary of $M^{\prime}$, such that $\partial M^{\prime}=\partial \mathcal{O}^{\prime}$. Let $\mathcal{Q}$ be a closed subset of $M$ such that the differential structure of $M$ induces an $n$-dimensional manifold with boundary structure on $\mathcal{Q}$. Suppose that there exists an isometry $\psi: \mathcal{O}^{\prime} \rightarrow \mathcal{Q}$. Define $\hat{M}=\left(M \cup M^{\prime}\right) / \psi$, i.e., we identify points $x, x^{\prime}$ in the union of $M$ and $M^{\prime}$ if and only if $x \in \mathcal{Q}, x^{\prime} \in \mathcal{O}^{\prime}$ and $\psi\left(x^{\prime}\right)=x$. Then $\hat{M}$ has the natural structure of a (Hausdorff) manifold without boundary and the spacetime $\left(\hat{M}, \hat{g}_{a b}\right)$ is an extension of $\left(M, g_{a b}\right)$, where $\hat{g}_{a b}$ is the metric on $\hat{M}$ naturally induced from $g_{a b}$ and $g_{a b}^{\prime}$. 
Proof: Consider the charts on $\hat{M}$ which arise either from charts on $M$ or charts on $\operatorname{int}\left(M^{\prime}\right)$. Then it is straightforward to verify that these charts comprise a compatible family which cover $\hat{M}$, thereby giving $\hat{M}$ the structure of a manifold without boundary. The Hausdorff property is obvious except for pairs of points in $\hat{M}$ arising from points $\left(x^{\prime}, x\right)$ with $x^{\prime}$ in the closure of $\mathcal{O}^{\prime}$ in $M^{\prime}$ and $x$ in the closure of $\mathcal{Q}$ in $M$. However, since by hypothesis $\mathcal{Q}$ is closed in $M, x$ is the image under $\psi$ of a point in $M^{\prime}$, from which it follows immediately that $x$ can be Hausdorff separated from $x^{\prime}$. It is straightforward to verify that $\left(\hat{M}, \hat{g}_{a b}\right)$ is an extension of $\left(M, g_{a b}\right)$.

We now state and prove our main result:

Theorem 4.2: Let $\left(M, g_{a b}\right)$ be a spacetime of the class specified at the end of section 2. Suppose, further, that surface gravity, $\kappa$, is a non-zero constant on the horizon $\mathcal{N}$. Then $\left(M, g_{a b}\right)$ can be extended globally so that in the enlarged spacetime the image of $\mathcal{N}$ will be a proper subset of a bifurcate Killing horizon. Furthermore, an extension $\left(M^{*}, g_{a b}^{*}\right)$ can be chosen so that the original one-parameter group of isometries $\chi_{u}$ extends to $\left(M^{*}, g_{a b}^{*}\right)$, and such that $\left(M^{*}, g_{a b}^{*}\right)$ possesses a "wedge reflection" isometry, $w$, about the bifurcation surface $S$.

Proof: Let $\left(\tilde{M}, \tilde{g}_{a b}\right)$ be the local extension of $\left(M, g_{a b}\right)$ constructed above. Let $\varepsilon$ be any positive function on $\sigma$ such that at each $\left(x^{1}, x^{2}\right)$, we have $\varepsilon\left(x^{1}, x^{2}\right)<\tilde{\varepsilon}_{(i)}\left(x^{1}, x^{2}\right)$ for some $i$. Let $M^{\prime}$ be the submanifold with boundary of $\tilde{M}$ consisting of the points of $\tilde{M}$ which satisfy

$$
|U V| \begin{cases}\leq \varepsilon\left(x^{1}, x^{2}\right), & \text { if } U>0 \\ <\varepsilon\left(x^{1}, x^{2}\right), & \text { if } U \leq 0\end{cases}
$$

Let $\mathcal{O}^{\prime}$ be the subset of $M^{\prime}$ satisfying $U>0$. Let $\mathcal{Q}$ be the pre-image of $\mathcal{O}^{\prime}$ under the isometric embedding map $\phi$ provided by the above local extension. It follows directly from lemma 3.4 that $\mathcal{Q}$ is a closed subset of $M$. Hence, the conditions of lemma 4.1 hold. Thus, we obtain a global extension, $\left(\hat{M}, \hat{g}_{a b}\right)$, of $\left(M, g_{a b}\right)$ by "patching it" to $\left(M^{\prime}, g_{a b}^{\prime}\right)$ in the manner explained in lemma 4.1.

Now, consider the new spacetime $\left(\hat{M}^{\prime}, \hat{g}_{a b}^{\prime}\right)$ obtained from $\left(\hat{M}, \hat{g}_{a b}\right)$ by reversing its time orientation and also eliminating the points which came from points in $\left(\tilde{M}, \tilde{g}_{a b}\right)$ satisfying both $U<0$ and $|U V|>\varepsilon\left(x^{1}, x^{2}\right) / 2$. Introduce new coordinates $\left(U^{\prime}, V^{\prime}\right)$ in a neighborhood of the bifurcate Killing horizon of $\left(\hat{M}^{\prime}, \hat{g}_{a b}^{\prime}\right)$ by $U^{\prime}=-U, V^{\prime}=-V$. Since $\left(\tilde{M}, \tilde{g}_{a b}\right)$ possesses the reflection isometry $U \rightarrow-U, V \rightarrow-V$ about the bifurcation surface, it is clear that the construction of the previous paragraph now can be repeated, with $\left(M^{\prime}, g_{a b}^{\prime}\right)$ replaced by 
$\left(\hat{M}^{\prime}, \hat{g}_{a b}^{\prime}\right)$. The isometries $\chi_{u}$ clearly extend to the resulting extension, which we denote as $\left(M^{*}, g_{a b}^{*}\right)$, and $\left(M^{*}, g_{a b}^{*}\right)$ manifestly also possesses a "wedge reflection" isometry about the bifurcation surface $U=V=0$.

We conclude this section with the following proposition, which shows that the extension $\left(M^{*}, g_{a b}^{*}\right)$ satisfies all of the properties needed for the application of the results of [8].

Proposition 4.3: Let $\left(M^{*}, g_{a b}^{*}\right)$ be the "wedge reflection" invariant extension of $\left(M, g_{a b}\right)$ constructed in theorem 4.2. Let $\mathcal{D}=M \backslash \mathcal{B}$ denote the domain of outer communications of $\left(M, g_{a b}\right)$. Then there exists a globally hyperbolic open region $\mathcal{V} \subset M^{*}$ which is invariant under the isometries $\chi_{u}$ such that $\overline{\mathcal{D}} \subset \mathcal{V}$, where $\overline{\mathcal{D}}$ denotes the closure of $\mathcal{D}$ in $M^{*}$.

Proof: Let $\mathcal{C}$ be a smooth, spacelike Cauchy surface for $\left(M, g_{a b}\right)$. Let $\tilde{\mathcal{C}}$ be the hypersurface in $\left(M, g_{a b}\right)$ obtained by removing the portion of $\mathcal{C}$ lying in $\mathcal{B}$ and replacing it with the portion of $\mathcal{N}$ lying in the causal past of $\sigma=\mathcal{N} \cap \mathcal{C}$. Finally, let $\mathcal{C}^{*}$ be the hypersurface in $\left(M^{*}, g_{a b}^{*}\right)$ obtained by adjoining to $\tilde{\mathcal{C}}$ the bifurcation surface, $S$, together with the image of $\tilde{\mathcal{C}}$ under the wedge reflection isometry, $w$. Then $\mathcal{C}^{*}$ is a closed, achronal, edgeless set. Let $p \in \mathcal{D}$. If $p \in J^{+}[\mathcal{C}]$, then any past inextendible causal curve through $p$ intersects $\mathcal{C}$ outside of $\mathcal{B}$, and, hence, must intersect $\mathcal{C}^{*}$. On the other hand, if $p \in J^{-}[\mathcal{C}]$, then any future-inextendible causal curve $\gamma$ must intersect $\mathcal{C}$. If $\gamma$ intersects $\mathcal{C}$ in $\mathcal{D}$, then clearly it intersects $\mathcal{C}^{*}$. But if $\gamma$ intersects $\mathcal{C}$ in $\mathcal{B}$, then $\gamma$ must cross $\mathcal{N}$ to the causal past of $\sigma$, and, hence, $\gamma$ also must intersect $\mathcal{C}^{*}$. Since $\mathcal{D}$ is open, this establishes that $\mathcal{D} \subset \mathcal{V}$, where $\mathcal{V} \equiv \operatorname{int} D\left[\mathcal{C}^{*}\right]$ and $D$ denotes the domain of dependence. Thus, in order to show that $\overline{\mathcal{D}} \subset \mathcal{V}$, it suffices to show that there cannot exist a point $p \in \partial \mathcal{D}$ such that $p \in \partial \mathcal{V}=\partial D\left[\mathcal{C}^{*}\right]$. However, if $p \in \partial \mathcal{D}$, then $p$ must lie either on the portion, $\mathcal{H}^{+}$, defined by $V=0, U \geq 0$ or the portion, $\mathcal{H}^{-}$, defined by $U=0, V \leq 0$, of the bifurcate Killing horizon of $\left(M^{*}, g_{a b}^{*}\right)$. In the former case, we have $p \in J^{+}\left[\mathcal{C}^{*}\right]$, so if $p \in \partial D\left[\mathcal{C}^{*}\right]$, we must have $p \in H^{+}\left[\mathcal{C}^{*}\right]$, where $H^{+}$denotes the future Cauchy horizon. However, in that case, $p$ must lie on a past inextendible null geodesic which does not intersect $\mathcal{C}^{*}$. But if $p \in \mathcal{H}^{+}$, then either $p \in \mathcal{C}^{*}$ - in which case every null geodesic through $p$ clear intersects $\mathcal{C}^{*}-$ or $p \in I^{+}\left[\mathcal{C}^{*}\right]$. In the latter case, every past inextendible causal curve through $p$ either enters $\mathcal{D}$ to the future of $\mathcal{C}^{*}-$ in which case, by the above argument, it intersects $\mathcal{C}^{*}-$ or it coincides with the null geodesic generator of the horizon - in which case it also intersects $\mathcal{C}^{*}$. The proof for the case $p \in \mathcal{H}^{-}$ 
proceeds similarly.

Finally, we note that $D\left[\mathcal{C}^{*}\right]$ may be characterized by the fact that $q \in D\left[\mathcal{C}^{*}\right]$ if and only if every inextendible causal curve through $q$ enters either $\overline{\mathcal{D}}$ or its image under the wedge reflection isometry, $w$. However, since $\overline{\mathcal{D}}$ is invariant under $\chi_{u}$, it follows that $D\left[\mathcal{C}^{*}\right]$ and, hence, $\mathcal{V}=\operatorname{int} D\left[\mathcal{C}^{*}\right]$ also is invariant under $\chi_{u}$.

\section{Extensions of matter fields}

In this section, we analyze the extendibility of "matter fields" defined on a spacetime $\left(M, g_{a b}\right)$ in the class we are considering. More precisely, let $T^{a_{1} \ldots a_{k}} b_{1} \ldots b_{l}$ be an arbitrary, smooth tensor field of type $(k, l)$ on $\left(M, g_{a b}\right)$ which is invariant under the isometries $\chi_{u}$, so that

$$
\mathcal{L}_{\xi} T^{a_{1} \ldots a_{k}} b_{1} \ldots b_{l}=0
$$

Under what conditions can $T^{a_{1} \ldots a_{k}} b_{1} \ldots b_{l}$ be extended to a smooth, isometry invariant tensor field on the extension, $\left(M^{*}, g_{a b}^{*}\right)$ constructed in theorem 4.2? For a tensor field of type $(0,0)$ (i.e., a function), it is straightforward to verify that isometry invariance on $M$ implies extendibility to $M^{*}$. However, this result does not hold for tensor fields of higher type. For example, in a region covered by EF coordinates the one-form $d u$ is smooth and isometry invariant, but cannot be smoothly extended to $M^{*}$, as can be seen from the fact that in terms of the Kruskal coordinates, we have $d u=d U / U$.

Since the spacetime metric extends smoothly to $\left(M^{*}, g_{a b}^{*}\right)$, without loss of generality it suffices to consider the case of tensor fields of type $(0, l)$. In the following, we shall supress indices and denote such a tensor field by $T$. The only obstruction to a smooth extension of $T$ will arise from the behavior of $T$ near the bifurcate Killing horizon, so without any loss of generality, we may restrict attention to the region of $\left(M^{*}, g_{a b}^{*}\right)$ where the Kruskal coordinates $(U, V)$ are well defined. Without loss of generality, we may also restrict attention to a subregion covered by a single patch of the coordinates $\left(x^{1}, x^{2}\right)$. Let $\mathcal{O}$ denote such a subregion. In addition, it will be useful, initially, to further restrict attention to the ("right wedge") portion, $\mathcal{R}$, of $\mathcal{O}$ consisting of points satisfying $U>0, V<0$. Thus, $\mathcal{R} \subset \mathcal{D}$, where $\mathcal{D}$ denotes the domain of outer communications of $M$. Our first lemma is the following:

Lemma 5.1: Within $\mathcal{R}$, the one-forms $d U / U, d V / V, d x^{1}$, and $d x^{2}$ are invariant under the isometries $\chi_{u}$ and are linearly independent at each point. 
Proof: The linear independence of $d U / U, d V / V, d x^{1}$, and $d x^{2}$ in $\mathcal{R}$ follows immediately from the linear independence of the Kruskal coordinate basis $d U, d V, d x^{1}$, and $d x^{2}$. Isometry invariance of $d x^{1}$, and $d x^{2}$ follows immediately from the isometry invariance of the EF coordinates $\left(x^{1}, x^{2}\right)$. Isometry invariance of $d U / U$ follows from the fact that the $\mathrm{EF}$ coordinate basis element $d u$ is isometry invariant, and from the definition (13) of $U$, we have $d U / U=d u$. Finally, the definitions of $U$ and $V$ immediately imply that $U V$ can be expressed as a function of $\left(r, x^{1}, x^{2}\right)$, and, hence is isometry invariant. Hence, $d(U V) /(U V)=d U / U+d V / V$ is isometry invariant, which, in turn, implies that $d V / V$ is isometry invariant.

Given a tensor field $T$ of type $(0, l)$ in $\mathcal{R}$, we expand it in the basis constructed from $d U / U$, $d V / V, d x^{1}$, and $d x^{2}$. Since this basis is isometry invariant, the tensor field $T$ will be isometry invariant if and only if all of the functions appearing in the basis expansion are themselves isometry invariant. This, in turn, is equivalent to requiring that these basis expansion functions can be expressed as functions of $\left(U V, x^{1}, x^{2}\right)$ only. Thus, in $\mathcal{R}$, the basis expansion of any isometry invariant tensor field, $T$, of type $(0, l)$ takes the schematic form

$$
T=\sum f^{(p),(q),(r),(s)}\left(U V, x^{1}, x^{2}\right)(d U / U)^{(p)}(d V / V)^{(q)}\left(d x^{1}\right)^{(r)}\left(d x^{2}\right)^{(s)},
$$

where the superscripts $p, q, r, s$ (with $p+q+r+s=l$ ) denote the number of times the basis element appears in the particular term in the expansion. Since $d U, d V, d x^{1}$, and $d x^{2}$ remain smooth and linearly independent in the extended spacetime, it follows that $T$ is extendible if and only if each term in the above basis expansion is separately extendible. The next lemma then follows immediately by inspection of the form of this basis expansion

Lemma 5.2: Let $T$ be a smooth, isometry invariant tensor field in $\mathcal{R}$. Then $T$ is smoothly extendible to the hypersurface $V=0, U>0$ in $M$ (i.e., to the original Killing horizon $\mathcal{N}$ ) if and only if each basis expansion function $f^{(p),(q),(r),(s)}$ can be written as

$$
f^{(p),(q),(r),(s)}\left(U V, x^{1}, x^{2}\right)=(U V)^{q} \alpha^{(p),(q),(r),(s)}\left(U V, x^{1}, x^{2}\right),
$$

where $\alpha^{(p),(q),(r),(s)}$ is a smooth function of its arguments. Similarly, $T$ is smoothly extendible to the hypersurface $U=0, V<0$ in $M^{*}$ if and only if each $f^{(p),(q),(r),(s)}$ can be written as

$$
f^{(p),(q),(r),(s)}\left(U V, x^{1}, x^{2}\right)=(U V)^{p} \beta^{(p),(q),(r),(s)}\left(U V, x^{1}, x^{2}\right),
$$


where $\beta^{(p),(q),(r),(s)}$ is smooth. Finally, $T$ is smoothly extendible as an isometry invariant tensor field to a neighborhood of the entire bifurcate Killing horizon $U V=0$ (and, hence, to all of $M^{*}$ ) if and only if

$f^{(p),(q),(r),(s)}\left(U V, x^{1}, x^{2}\right)=(U V)^{\max (p, q)} \gamma^{(p),(q),(r),(s)}\left(U V, x^{1}, x^{2}\right)$,

where $\gamma^{(p),(q),(r),(s)}$ is smooth.

If $T$ is given as a smooth tensor field on $\left(M, g_{a b}\right)$, then we know that as a tensor field on $\mathcal{R}$ it is smoothly extendible to $\mathcal{N}$. Thus, each $f^{(p),(q),(r),(s)}$ must take the form

$$
f^{(p),(q),(r),(s)}\left(U V, x^{1}, x^{2}\right)=(U V)^{q} \alpha^{(p),(q),(r),(s)}\left(U V, x^{1}, x^{2}\right) .
$$

It then follows immediately from lemma 5.2 that the necessary and sufficient condition for the extendibility of $T$ to an isometry invariant tensor field on $\left(M^{*}, g_{a b}^{*}\right)$ is that for all terms in the basis expansion with $p>q$ we have

$$
\alpha^{(p),(q),(r),(s)}\left(U V, x^{1}, x^{2}\right)=(U V)^{p-q} \tilde{\alpha}^{(p),(q),(r),(s)}\left(U V, x^{1}, x^{2}\right),
$$

where $\tilde{\alpha}^{(p),(q),(r),(s)}$ is smooth.

Our final result is the following:

Theorem 5.3: Let $\left(M, g_{a b}\right)$ be a spacetime in the class specified at the end of section 2 , and suppose, furthermore, that $\left(M, g_{a b}\right)$ either is static or is stationary-axisymmetric with a " $t-\phi$ " reflection isometry. Let $i$ denote, respectively, the " $t$ " or " $t-\phi$ " reflection isometry in $\mathcal{D}$. Let $T$ be a smooth tensor field on $\left(M, g_{a b}\right)$ which is invariant under the isometries $\chi_{u}$ and also is invariant under $i$ in $\mathcal{D}$. Then $T$ is smoothly extendible to an isometry invariant tensor field on $\left(M^{*}, g_{a b}^{*}\right)$.

Proof: Consider the restriction of $T$ to the region, $\mathcal{R}$, of $\left(M, g_{a b}\right)$ defined above. Since $T$ is given as smooth on $\left(M, g_{a b}\right)$, it obviously is smoothly extendible to $\mathcal{N}$ given by $V=0, U>0$. However, the isometry $i$ extends to the boundary of $\mathcal{R}$ in $\left(M^{*}, g_{a b}^{*}\right)$ in such a way as to map the hypersurface $V=0, U>0$ into the hypersurface $U=0, V<0$. Since $T$ is invariant under $i$, it follows that $T$ must also be extendible to the hypersurface $U=0, V<0$. It then follows from lemma 5.2 that $T$ is extendible to $\left(M^{*}, g_{a b}^{*}\right)$. 


\section{Acknowledgements}

This research was supported in part by NSF grant PHY 92-20644, OTKA grants F14196, T016246. One of us (IR) wishes to thank to the Fermi Institute for its hospitality during a visit, which was made possible by a supplement to grant PHY 92-20644 provided by the International Division of the NSF and MTA (Hungarian Academy of Sciences) grant 77-94 for scientific collaborations.

\section{References}

[1] R.M. Wald : General Relativity (University of Chicago Press, 1984)

[2] S.W. Hawking: Commun. Math. Phys. 25, 152-166 (1972)

[3] S.W. Hawking and G.R.F. Ellis: Large Scale Structure of Spacetime (Cambridge University Press, Cambridge, 1973)

[4] B. Carter: Phys. Rev. Lett. 26, 331-333 (1971)

[5] B. Carter: in Black holes, ed. C. DeWitt and B.S. Dewitt (New York: Gordon \& Breach, 1973)

[6] I. Rácz and R.M. Wald: Class. Quant. Grav. 9, 2643-2656 (1992)

[7] I. Rácz: J. Math Phys. 34, 2448-2464 (1993)

[8] B.S. Kay and R.M. Wald: Phys. Rep. 207, 49-136 (1991)

[9] M. Heusler: Black Hole Uniqueness Theorems, Cambridge Lecture Notes in Physics (Cambridge, in press)

[10] P.T. Chruściel and R.M. Wald: Common. Math. Phys. 163, 561-604 (1994) 